\title{
GHEORGHE PĂUN
}

\section{On simple matrix languages versus scattered context languages}

RAIRO - Informatique théorique, tome $16, \mathrm{n}^{\circ} 3$ (1982), p. 245-253.

<http://www.numdam.org/item?id=ITA_1982_16_3_245_0>

(C) AFCET, 1982, tous droits réservés.

L'accès aux archives de la revue « RAIRO - Informatique théorique » implique l'accord avec les conditions générales d'utilisation (http://www.numdam. org/legal.php). Toute utilisation commerciale ou impression systématique est constitutive d'une infraction pénale. Toute copie ou impression de ce fichier doit contenir la présente mention de copyright.

\section{Numdam}

Article numérisé dans le cadre du programme

Numérisation de documents anciens mathématiques

http://www.numdam.org/ 


\title{
ON SIMPLE MATRIX LANGUAGES VERSUS SCATTERED CONTEXT LANGUAGES $\left({ }^{*}\right)$
}

\author{
by Gheorghe PĂlN $\left({ }^{1}\right)$ \\ Communicated by J. BerSTEL
}

\begin{abstract}
We prove that the family of simple matrix languages of Ibarra is strictly included in the family of scattered context languages of Greibach and Hopcroft.

Résumé. - Nous prouvons que la famille des langages simplement matriciels de Ibarra est strictement contenue dans la famille des «scattered context " langages de Greibach et Hopcroft.
\end{abstract}

\section{INTRODUCTION}

In formal language theory, many restrictions in the derivation of context-free grammars were introduced in order to increase the generative capacity of these grammars. Despite the great attention paid to the relationships between different such restrictions, there still are many open problems in this area. The present paper aims to clarify the relation between two known and important restrictions, namely, the simple matrix grammars of Ibarra [4] and the scattered context grammars of Greibach and Hopcroft [2]. We prove that each simple matrix language is a scattered context one, but the converse is not true. The result is an expected one taking into account the large generative capacity of scattered context grammars [2].

\section{DEFINITIONS}

Following [4], a simple matrix grammar of order $n$ is an $(n+3)$-tuple $G=\left(V_{1}, V_{2}, \ldots, V_{n}, \Sigma, S, M\right)$, where:

(a) $V_{1}, \ldots, V_{n}, \Sigma$ are mutually disjoint nonempty vocabularies, the elements

(*) Received in January 1980, revised in January 1981.

( $\left.{ }^{1}\right)$ Institute of Mathematics, Str. Academiei 14, Bucuresti R-70109 Romania.

R.A.I.R.O. Informatique théorique/Theoretical Informatics, 0399-0540/1982/245/\$ 5.00 AFCET-Bordas-Dunod 
of the set $V_{N}=\bigcup_{i=1}^{n} V_{i}$ being called nonterminals and the elements of $\Sigma$ being called terminals;

(b) $S \notin V_{N} \cup \Sigma$ is the start symbol of the grammar;

(c) $M$ is a finite set of matrices of the form $\left(S \rightarrow x_{1} x_{2} \ldots x_{n}\right)$ or of the form $\left(A_{1} \rightarrow x_{1}, \ldots, A_{n} \rightarrow x_{n}\right), A_{i} \in V_{i}, x_{i} \in\left(V_{i} \cup \Sigma\right)^{*}, i=1,2, \ldots, n$, and the number of nonterminals in $x_{i}$ (denoted by $N\left(x_{i}\right)$ ) is equal to the number of nonterminals in $x_{j}$ for all values of $i, j$. (For a vocabulary $V$ we denote by $V^{*}$ the free monoid generated by $V$ under the operation of concatenation and the null element $\lambda$.) The vector of the $j$-th nonterminals of each $x_{i}$ as above is denoted by $\operatorname{poz}_{j}\left(x_{1}, \ldots, x_{n}\right)$.

For $y_{i}, z_{i} \in\left(V_{i} \cup \Sigma\right)^{*}, i=1,2, \ldots, n$, one writes $y_{1} y_{2} \ldots y_{n} \Rightarrow z_{1} z_{2} \ldots z_{n}$ iff $y_{i}=u_{i} A_{i} v_{i}, z_{i}=u_{i} x_{i} v_{i}, u_{i} \in \Sigma^{*}, v_{i} \in\left(V_{i} \cup \Sigma\right)^{*}$ for each $i=1,2, \ldots, n$, and $\left(A_{1} \rightarrow x_{1}, \ldots, \quad A_{n} \rightarrow x_{n}\right) \in M$. Also, we write $S \Rightarrow x_{1} x_{2} \ldots x_{n}$ iff $\left(S \rightarrow x_{1} x_{2} \ldots x_{n}\right) \in M$. The language generated by the grammar $G$ is $L(G)=\left\{\dot{w} \in \Sigma^{*} \mid S \stackrel{*}{\Rightarrow} w\right\}$ where $\stackrel{*}{\Rightarrow}$ is the reflexive transitive closure of $\Rightarrow$. We denote by $\mathscr{S} \mathscr{M}(n)$ the family of languages generated by simple matrix grammars of order $n$ and by $\mathscr{S} \mathscr{M}$ the family of all simple matrix languages.

In words, a simple matrix grammar has the nonterminal vocabulary partitioned into $n$ disjoint sets and the $i$-th rules in its matrices contain only nonterminals in the $i$-th set of this partition. Moreover, the rules are used in the leftmost manner for the sentential form subwords containing nonterminals in the corresponding set.

Example: Let $G=\left(V_{N}, \Sigma, S, P\right)$ be a context-free grammar and let $c$ be a symbol not in $\Sigma$. The language

$$
D(L(G))=\{x c x \mid x \in L(G)\}
$$

is not context-free for all $G$, but it can be generated by the simple matrix grammar of order 2 :

$$
G^{\prime}=\left(V_{1}, V_{2}, \Sigma \cup\{c\}, S, M\right)
$$

where

$$
V_{1}=\left\{A^{\prime} \mid A \in V_{N}\right\}, V_{2}=\left\{A^{\prime \prime} \mid A \in V_{N}\right\}
$$

and $M=\left\{\left(S \rightarrow S^{\prime} c S^{\prime \prime}\right)\right\} \cup\left\{\left(A^{\prime} \rightarrow x^{\prime}, A^{\prime \prime} \rightarrow x^{\prime \prime}\right) \mid A \rightarrow x\right.$ is a rule in $P$ and $x^{\prime}, x^{\prime \prime}$ are obtained by replacing all nonterminal symbols $B$ in $x$ by $B^{\prime}$, respectively, by $\left.B^{\prime \prime}\right\}$. Indeed, the leftmost derivation "on $V_{1}$ and on $V_{2}$ " ensures the equality of the strings generated in the left and in the right hand side of the symbol $c$.

R.A.I.R.O. Informatique théorique/Theoretical Informatics 
According to [2], a scattered context grammar is a quadruple $G=\left(V_{N}, \Sigma, S, M\right)$, where $V_{N}, \Sigma$ are finite nonempty disjoint vocabularies $\left(V_{N}\right.$ is the nonterminal and $\Sigma$ is the terminal vocabulary), $S \in V_{N}$ is the start symbol and $M$ is a finite set of matrix rules of the form $\left(A_{1} \rightarrow x_{1}, \ldots, A_{n} \rightarrow x_{n}\right), n \geqq 1, A_{i} \in V_{N}$, $x_{i} \in\left(V_{N} \cup \Sigma\right)^{*}$. If $z=z_{1} A_{1} z_{2} \ldots z_{n} A_{n} z_{n+1}, \quad w=z_{1} x_{1} z_{2} x_{2} \ldots z_{n} x_{n} z_{n+1}$, $z_{i} \in\left(V_{N} \cup \Sigma\right)^{*}$ for each $i$ and $\left(A_{1} \rightarrow x_{1}, \ldots, A_{n} \rightarrow x_{n}\right) \in M$, then we write $z \Rightarrow w$. The language generated by the grammar $G$ is $L(G)=\left\{x \in \Sigma^{*} \mid S \stackrel{*}{\Rightarrow} x\right\}$. Let $\mathscr{S}$ be the family of languages generated by $\lambda$-free scattered context grammars.

The key feature of the scattered context grammars is the matrices use manner: the rules of a matrix replace occurrences of their left-hand sides in the order in which the rules appear in that matrix.

Example: Let us consider a language $L \subseteq V^{*}$. For $x, y \in V^{*}$ we define:

$$
\begin{gathered}
\text { Shuf }(x, y)=\left\{x_{1} y_{1} x_{2} y_{2} \ldots x_{n} y_{n} \mid n \geqq 1,\right. \\
\left.x_{i}, y_{i} \in V^{*}, x=x_{1} \ldots x_{n}, y=y_{1} \ldots y_{n}\right\} .
\end{gathered}
$$

For $L_{1}, L_{2} \subseteq V^{*}$ we put:

$$
\operatorname{Shuf}\left(L_{1}, L_{2}\right)=\bigcup_{\substack{x \in L_{1} \\ y \in L_{2}}} \operatorname{Shuf}(x, y) .
$$

and we define:

$$
\text { Shuf* }(L)=\bigcup_{n=1}^{\infty} \operatorname{Shuf}^{n}(L),
$$

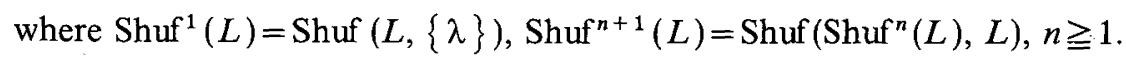

For any finite language $L$, the language Shuf* $(L)$ is in the family $\mathscr{S}$. Indeed, for given $L$, the scattered context grammar $G=(\{S\}, V, S, M)$ with $M=\{(\mathrm{S} \rightarrow \mathrm{SS})\} \cup\left\{\left(\mathrm{S} \rightarrow \mathrm{a}_{1}, S \rightarrow a_{2}, \ldots, S \rightarrow a_{n}\right) \mid n \geqq 1, a_{i} \in V, a_{1} a_{2} \ldots a_{n} \in L\right\}$, clearly generates the language Shuf* $(L)$. (For simple languages $L$, the language Shuf* $(L)$ is not context free. For example, Shuf* $(\{a b c\}) \cap\left\{a^{n} b^{m} c^{p} \mid n, m\right.$, $p \geqq 1\}=\left\{a^{n} b^{n} c^{n} \mid n \geqq 1\right\}$ hence Shuf* $(\{a b c\})$ is not a context-free language although $\{a b c\}$ is a singleton.)

\section{EACH SIMPLE MATRIX LANGUAGE IS A SCATTERED CONTEXT LANGUAGE}

In [4] one claims, without proof, that each simple matrix language is a matrix language. We feel that this does not hold. For instance, we believe that there are vol. $16, n^{\circ} 3,1982$ 
context-free languages $L$ for which the language $D(L)$ defined as in the above first example is not a matrix one (see similar arguments in [6]). Moreover, in [4] it is shown that the family $\mathscr{S} \mathscr{M}$ is included in the family of deterministic contextsensitive languages. As the relation between deterministic context-sensitive languages and the scattered context languages is an open problem, the relation between the families $\mathscr{S} \mathscr{M}$ and $\mathscr{S}$ is a significant question.

The problem is answered below. We shall prove that the family $\mathscr{S} \mathscr{M}$ is strictly included in $\mathscr{S}$. A lemma proved in [5] is needed in this aim.

A simple matrix grammar is called purely leftmost iff for any matrix $\left(A_{1} \rightarrow x_{1}, \ldots, A_{n} \rightarrow x_{n}\right)$ in $M$ and for any $w_{1}, w_{2}, \ldots, w_{n}$ such that $S \stackrel{*}{\Rightarrow} u_{1} w_{2} \ldots w_{n}, w_{i} \in\left(V_{i} \cup \Sigma\right)^{*}$, we have either :

$$
\operatorname{poz}_{1}\left(w_{1}, \ldots, w_{n}\right)=\left(\mathrm{A}_{1}, \ldots, \mathrm{A}_{n}\right)
$$

or :

$$
\operatorname{poz}_{1}\left(w_{1}, \ldots, w_{n}\right)=\left(B_{1}, \ldots, B_{n}\right), A_{i} \neq B_{i} \text { for all } i=1,2, \ldots, n .
$$

LEMMA 1 [5]: For any simple matrix grammar $G$ there is a purely leftmost simple matrix grammar $G^{\prime}$ such that $L(G)=L\left(G^{\prime}\right)$.

On the other hand, by extending to simple matrix grammars the arguments used for eliminating the rules of the form $A \rightarrow \lambda$ and $A \rightarrow B$ from context-free grammars [9], one can easily see that for any simple matrix grammar $G$ there is an equivalent simple matrix grammar $G^{\prime}$ which does not contain matrices of the form $\left(A_{1} \rightarrow \lambda, \ldots, A_{n} \rightarrow \lambda\right)$ or of the form $\left(A_{1} \rightarrow B_{1}, \ldots, A_{n} \rightarrow B_{n}\right), A_{i}, B_{i} \in V_{i}$. This assertion can be also obtained as a consequence of the proof of Theorem 1.1 in [4]: by Lemma 1.2 [4], for each $L \in \mathscr{S} \mathscr{M}(n)$ we can construct an $n$-context-free language $L^{\prime} \leqq\left[V^{*}\right]^{n}$ such that $L=\left\{x_{1} \ldots x_{n} \mid\left(x_{1}, \ldots, x_{n}\right) \in L^{\prime}\right\}$ and $L^{\prime}$ is the image of a usual context-free language $L^{\prime \prime}$ by a certain operator $\mathscr{T}_{n}$. Giving a context-free grammar $G$ for $L^{\prime \prime}$ which does not contain rules of the form $A \rightarrow \lambda$, $A \rightarrow B$, by means of the construction in Lemma 1.1 [4], we can get a simple matrix grammar of order $n$ for the language $L$ which does not contain matrices of the form $\left(A_{1} \rightarrow \lambda, \ldots, A_{n} \rightarrow \lambda\right),\left(A_{1} \rightarrow B_{1}, \ldots, A_{n} \rightarrow B_{n}\right)$.

We shall prove the inclusion $\mathscr{S} \mathscr{M} \cong \mathscr{S}$ in two phases: first we shall reduce the problem to the inclusion $\mathscr{S} \mathscr{M}(2) \subseteq \mathscr{S}$, then we shall simulate the leftmost derivation in a simple matrix grammar of order 2 by means of matrices in a scattered context grammar.

LemMa 2: Any language in $\mathscr{S} \mathscr{M}(n), n$ given, is the homomorphic image of the intersection of $n$ languages in $\mathscr{S} \mathscr{M}(2)$.

R.A.I.R.O. Informatique théorique/Theoretical Informatics 
Proof: Let $G=\left(V_{1}, \ldots, V_{n}, \Sigma, S, M\right)$ be a purely leftmost simple matrix grammar of order $n$ and let $m_{1}, \ldots, m_{r}$ be distinct labels associated to the matrices in $M$. Clearly, in a purely leftmost simple matrix grammar the control word describing a derivation precisely identifies that derivation. So, for each $i=1,2, \ldots, n$ we shall construct a simple matrix grammar of order 2 which generates the " $i$-th component" of a string in $L(G)$ together with the control word associated to this derivation (and some other arbitrary symbols). Intersecting such languages (which are of order 2), we shall obtain the strings in $L(G)$ together with the control words of the corresponding derivations (and some auxiliary symbols). Erasing the control word (and the auxiliary symbols) by a homomorphism, we shall get the language $L(G)$. Following these ideas, let us consider the grammars:

$$
G_{i}=\left(V_{1}^{i}, V_{2}^{i}, \Sigma \cup C, S, M_{i}\right), \quad i=1,2, \ldots, n,
$$

where:

$$
\begin{gathered}
V_{1}^{i}=V_{i} \cup\{X, Y\}, \quad V_{2}^{i}=\{Z\}, \\
C=\left\{c_{1}, \ldots, c_{n}, d_{1}, \ldots, d_{n}\right\},
\end{gathered}
$$

and $M_{i}$ is constructed in the following way:

(1) If $1<i<n$, then for each matrix $m_{j}:\left(S \rightarrow w_{1} w_{2} \ldots w_{n}\right)$ in $M$ we introduce in $M_{i}$ the matrix:

$$
\left(S \rightarrow X c_{i} w_{i} d_{i} Y m_{i} Z^{N\left(w_{i}\right)+2}\right) .
$$

(Remember that $N(w)$ is the number of nonterminal occurrences in the string $w$.)

If $i=1$, then we introduce the matrix:

$$
\left(S \rightarrow c_{1} w_{1} d_{1} Y m_{j} Z^{N\left(w_{1}\right)+1}\right),
$$

and if $i=n$, then we introduce the matrix:

$$
\left(S \rightarrow X c_{n} w_{n} d_{n} m_{j} Z^{N\left(w_{n}\right)+1}\right) .
$$

(2) The following matrices belong to $M_{i}$ for $1<i<n$ :

$$
\begin{aligned}
&(X \rightarrow a X, Z \rightarrow Z), \quad a \in \Sigma \cup\left\{c_{1}, \ldots, c_{i-1}, d_{1}, \ldots, d_{i-2}\right\}, \\
&\left(X \rightarrow d_{i-1}, Z \rightarrow \lambda\right), \\
&(Y \rightarrow a Y, Z \rightarrow Z), \quad a \in \Sigma \cup\left\{c_{i+1}, \ldots, c_{n}, d_{i+1}, d_{n-1}\right\}, \\
& \quad\left(Y \rightarrow d_{n}, Z \rightarrow \lambda\right) .
\end{aligned}
$$

Moreover, for $i=1$ we introduce in $M_{i}$ the above matrices containing the symbol $Y$ and for $i=n$ we introduce the matrices containing the symbol $X$. 
(3) For each matrix $m_{j}:\left(A_{1} \rightarrow x_{1}, \ldots, A_{n} \rightarrow x_{n}\right)$ in $M$ we introduce in $M_{i}$ the matrix:

$$
\left(A_{i} \rightarrow x_{i}, Z \rightarrow m_{j} Z^{N\left(x_{i}\right)}\right)
$$

Obviously, we have:

$$
\begin{gathered}
L\left(G_{i}\right)=\left\{x c_{i} y d_{i} z w \mid x \in\left(\Sigma \cup\left\{c_{1}, \ldots, c_{i-1}, d_{1}, \ldots, d_{i-1}\right\}\right)^{*}, y \in \Sigma^{*},\right. \\
z \in\left(\Sigma \cup\left\{c_{i+1}, \ldots, c_{n}, d_{i+1}, \ldots, d_{n}\right\}\right)^{*}, w \in\left\{m_{1}, \ldots, m_{r}\right\}^{*}, \\
w \text { is the control word associated to the derivation } \\
\text { of } \left.y \text { according to the grammar } G_{i}\right\} .
\end{gathered}
$$

As the grammar $G$ is a purely leftmost one, the control word $w$ precisely identifies the derivation and the string $y$, hence we have:

$$
\bigcap_{i=1}^{n} L\left(G_{i}\right)=\left\{c_{1} y_{1} d_{1} c_{2} y_{2} d_{2} \ldots c_{n} y_{n} d_{n} w \mid y_{1} y_{2} \ldots y_{n}\right.
$$

can be derived from $S$ in the grammar $G$

and $w$ is the control word associated to such a derivation $\}$.

Let us denote by $H$ the above language and let $h$ be the homomorphism which erases all symbols $c_{i}, d_{i}$ and $m_{j}$. We obtain the equation:

$$
L(G)=h(H)
$$

and the lemma is proved.

LEMMA 3: $\mathscr{S} \mathscr{M}(2) \subseteq \mathscr{S}$.

Proof: Let $G=\left(V_{1}, V_{2}, \Sigma, S, M\right)$ be a simple matrix grammar of order 2 without matrices containing two $\lambda$-rules. We construct the following scattered context grammar:

$$
G^{\prime}=\left(V_{N}, \Sigma \cup\{c\}, S, M^{\prime}\right)
$$

where:

$$
V_{N}=V_{1} \cup V_{2} \cup\left\{S, c^{\prime}\right\} \cup\left\{a^{\prime} \mid a \in V_{1} \cup V_{2} \cup \Sigma\right\}
$$

and the set $M^{\prime}$ is obtained in the following way:

For each matrix $(S \rightarrow w) \in M, w \in \Sigma^{*}$, we introduce in $M^{\prime}$ the matrix $(S \rightarrow \dot{w})$. and for each nonterminal matrix $(S \rightarrow a x b y) \in M, a \in V_{1} \cup \Sigma, x \in\left(V_{1} \cup \Sigma\right)^{*}$, $b \in V_{2} \cup \Sigma, y \in\left(V_{2} \cup \Sigma\right)^{*}$, we introduce the matrix $\left(S \rightarrow a^{\prime} x b^{\prime} y\right)$. 
For each matrix of the form $\left(A_{1} \rightarrow x_{1}, A_{2} \rightarrow x_{2}\right)$, we replace $x_{1}$ by $c$ if $x_{1}=\lambda$ and we replace $x_{2}$ by $c$ if $x_{2}=\lambda$. Let $\left(A_{1} \rightarrow b x, A_{2} \rightarrow e y\right)$ be the obtained matrix (the initial matrix if $\left.x_{1} \neq \lambda, x_{2} \neq \lambda\right), \quad b \in V_{1} \cup \Sigma \cup\{c\}, \quad x \in\left(V_{1} \cup \Sigma\right)^{*}$, $e \in V_{2} \cup \Sigma \cup\{c\}, y \in\left(V_{2} \cup \Sigma\right)^{*}$.

We introduce in $M^{\prime}$ the following matrices:

$$
\begin{gathered}
\left(a^{\prime} \rightarrow a, A_{1} \rightarrow b^{\prime} x, d^{\prime} \rightarrow d, A_{2} \rightarrow e^{\prime} y\right), a, d \in \Sigma \cup\{c\}, \\
\left(A_{1}^{\prime} \rightarrow b^{\prime} x, d^{\prime} \rightarrow d, A_{2} \rightarrow e^{\prime} y\right), d \in \Sigma \cup\{c\}, \\
\left(a^{\prime} \rightarrow a, A_{1} \rightarrow b^{\prime} x, A_{2}^{\prime} \rightarrow e^{\prime} y\right), a \in \Sigma \cup\{c\}, \\
\left(A_{1}^{\prime} \rightarrow b^{\prime} x, A_{2}^{\prime} \rightarrow e^{\prime} y\right) .
\end{gathered}
$$

We consider also the terminal matrices:

$$
\left(a^{\prime} \rightarrow a, b^{\prime} \rightarrow b\right), a, b \in \Sigma \cup\{c\} .
$$

Clearly, the grammar $G^{\prime}$ is a $\lambda$-free one. Let $h$ be the homomorphism which erases the letter $c$. We have:

$$
L(G)=h\left(L\left(G^{\prime}\right)\right)
$$

(Any sentential form according to the scattered context grammar $G^{\prime}$ contains exactly two primed symbols. The use of a matrix in $M^{\prime}$ is allowed only in the right hand side of these symbols. This restriction ensures the simulation of a leftmost application of the corresponding two-rules matrices in $M$. If a matrix is not used in the leftmost manner - that is, the primes do not circulate correctly - then the derivation cannot be terminated.)

As each matrix in $M$ contains at most a $\lambda$-rule, it follows that $|h(x)| \geqq 1 / 2|x|$. (We denoted by $|z|$ the length of the string $z$.) Consequently, $h$ is a linear erasing homomorphism. As the family $\mathscr{S}$ is closed under such homomorphisms [2], it follows that $h\left(L\left(G^{\prime}\right)\right) \in \mathscr{S}$, hence $L(G) \in \mathscr{S}$ and the lemma is proved.

Theorem: The family $\mathscr{S} \mathscr{M}$ is strictly included in the family $\mathscr{S}$.

Proof: According to the above lemmas, any language $L \in \mathscr{S} \mathscr{M}(n)$ can be written as:

$$
L=h\left(\bigcup_{i=1}^{n} L_{i}\right), \quad L_{i} \in \mathscr{S} \text { for each } i
$$

$h$ being the homomorphism in the proof of Lemma 2 . The family $\mathscr{S}$ is closed under intersection [2]. The homomorphism $h$ is a linear erasing one. Indeed, each nonterminal matrix in a simple matrix grammar increases the length of the 
rewritten string at least by one symbol and there are no $\lambda$-matrices. Thus, the homomorphism $h$ erases $2 n$ symbols $c_{i}, d_{i}$ and a control word shorter than the longest string derived between $c_{i}, d_{i}, i=1,2, \ldots, n$. As the family $\mathscr{S}$ is closed under linear erasing homomorphisms [2], it follows that $L \in \mathscr{S}$ hence $\mathscr{S} \mathscr{M} \subseteq \mathscr{S}$.

The inclusion is, obviously, proper. For instance, the language:

$$
L=\left\{a^{n} b^{n} c^{n} \mid n \geqq 1\right\}^{*},
$$

is not in $\mathscr{S} \mathscr{M}$ [4], but it is generated by the scattered context grammar:

$$
G=(\{S, A, B, C\},\{a, b, c\}, S, M),
$$

with:

$$
\begin{aligned}
M=\{(S \rightarrow A B C),(S \rightarrow \lambda),(S \rightarrow A B),(A \rightarrow a A b, B \rightarrow c B), & \\
(A \rightarrow a b, B \rightarrow c, C \rightarrow A B C),(A \rightarrow a b, B \rightarrow c), & (A \rightarrow a b, B \rightarrow c, C \rightarrow A B)\} .
\end{aligned}
$$

\section{FINAL REMARKS}

Let $\mathscr{S} \mathscr{M}_{f}$ and $\mathscr{S}_{f}$ be the families of finite index languages in $\mathscr{S} \mathscr{M}$, respectively, in $\mathscr{S}$. (See the index definition in [1].) Let us observe that the above proofs of Lemmas 2 and 3 do not modify the index finiteness. Consequently, $\mathscr{S} \mathscr{M}_{f} \subseteq \mathscr{S}_{f}$. The inclusion is proper since the language $\left\{a^{n} b^{n} c^{n} \mid n \geqq 1\right\}^{*}$ belongs to the family $\mathscr{S}_{f}$.

The inclusion $\mathscr{S} \mathscr{M}_{f} \subset \mathscr{S} \mathscr{M}$ is a proper one. Indeed, let us consider the context-free language $L=\{c\}(D\{c\})^{+}$, where $D$ is the Dyck language over the vocabulary $\{a, b\}$. As in [8] it was proved, $D$ has an infinite index according to the context-free grammars. Let us assume that $L \in \mathscr{S} \mathscr{M}_{f}$ and let $G=\left(V_{1}, \ldots, V_{n},\{a, b, c\}, S, M\right)$ be a simple matrix grammar of finite index generating the language $L$. Let $G_{i}$ be the context-free grammars $G_{i}=\left(V_{i} \cup\{S\},\{a, b, c\}, S, \quad\left\{S \rightarrow w_{i} \mid\left(S \rightarrow w_{1} w_{2} \ldots w_{n}\right) \in M\right.\right.$, $\left.w_{i} \in\left(V_{i} \cup\{a, b, c\}\right)^{*}\right\} \cup\left\{A_{i} \rightarrow x_{i} \mid\left(A_{1} \rightarrow x_{1}, \ldots, A_{n} \rightarrow x_{n}\right) \in M, A_{i} \in V_{i}\right.$, $\left.\left.x_{i} \in\left(V_{i} \cup\{a, b, c\}\right)^{*}\right\}\right)$. Let $g$ be a gsm which maps a string $w$ into $x$ providing that $w=y c x c z, x \in\{a, b\}^{*}, y, z \in\{a, b, c\}^{*}$. It is easy to see that we have $D=\bigcup_{i=1}^{n} g\left(L\left(G_{i}\right)\right)$. As $G$ is a finite index simple matrix grammar (we assume it to be purely leftmost), it follows that each grammar $G_{i}$ has a finite index too. The family of finite index context-free languages is a full-AFL [3]. It follows $D$ is a 
finite index context-free language. Contradiction with the result in [8]. Consequently, $L \in \mathscr{S} \mathscr{M}(1)-\mathscr{S} \mathscr{M}_{f}$.

From the above remarks it follows that the inclusion $\mathscr{S}_{\mathscr{M}_{f}} \subset \mathscr{S}_{f}$ does not imply $\mathscr{S} \mathscr{M}(k) \subset \mathscr{S}_{f}$, for some $k \geqq 1$. In fact, we feel that such an inclusion does not hold.

Open problem: Is the family $\mathscr{S} \mathscr{M}$ included in the family of languages generated by $\lambda$-free context-free matrix grammars in the appearance checking mode (See Chap. V in [9] or the monograph [7] for the theory of matrix grammars and languages.)

Note: Useful remarks by the referee are acknowledged, allowing us to make clearer some parts of the paper.

\section{REFERENCES}

1. B. Brainerd, An Analog of a Theorem about Context-Free Languages, Information and Control, Vol. 11, 1968, pp. 561-568.

2. S. Greibach and J. HopCroft, Scattered Context Grammars, J. of Computer and System Science, Vol. 3, 1969, pp. 232-247.

3. J. GRUSKA, A Few Remarks on the Index of Context-Free Grammars and Languages, Information and Control, Vol. 19, 1971, pp. 216-223.

4. O. Ibarra, Simple Matrix Languages, Information and Control, Vol. 17, 1970, pp. 259-294.

5. GH. PX̌un, On the Generative Capacity of Simple Matrix Grammars of Finité Index, Information Processing Letters, Vol. 7, No. 2, 1978, pp. 100-102.

6. GH. PǍn, On the Family of Finite Index Matrix Languages, J. of Computer and System Science, Vol. 18, 1979, pp. 267-280.

7. GH. PA̛u, Matrix Grammars, The Scientific and Enciclopaedic Publishing House, Bucharest, 1981 (in Romanian).

8. A. SalomaA, On the Index of Context-Free Languages, Information and Control, Vol. 14, 1969, pp. 474-477.

9. A. SalomaA, Formal Languages, Academic Press, New York and London, 1973. 